COMMENT. This report of an encephalitis lethargica (EL) syndrome, with similar characteristics to that of von Economo's EL first described in 1916, may now be added to the growing list of immune-mediated movement and neuropsychiatric disorders linked to group A streptococcal infections. Sydenham's chorea is the classic phenotype and more recently, pediatric autoimmune neuropsychiatric disorder (PANDAS) and post-streptococcal dystonia (Dale et al, 2001). That anti-basal ganglia antibodies are pathogenic in this spectrum of autoimmune disorders is suggested but not yet proven. Questions regarding the regional specificity of the antibodies, and the possibility that they may be markers for a destructive process, secondary to $\mathrm{T}$ cell-mediated or direct toxicity from the streptococcus infection, need to be clarified (Vincent A. Editorial comment. Brain 2004;127:2-3). A response of EL to plasma exchange or other immunotherapies (Blunt et al, 1997), as sometimes reported in PANDAS, would be of interest. Behavior disorders, resembling those of attention-deficithyperactivity and oppositional defiant disorders, and termed 'organic drivenness,' were described as complications of encephalitis following the influenza pandemic of 1918 (Hohman, Ebaugh, 1922, 1923; Kahn, Cohen, 1934).

\title{
CORONAVIRUS AND ADEM
}

A boy aged 15 years with acute disseminated encephalomyelitis (ADEM) found to have human coronavirus ( $\mathrm{HCoV}$ ) in the cerebrospinal fluid (CSF) and nasopharynx is reported from the Children's Hospital of Buffalo, SUNY at Buffalo, NY. The child presented with a 5-day history of numbness that began in the lower extremities and progressed to the umbilicus. He had difficulty in walking, clumsiness in the right hand, and increased irritability. An upper respiratory infection had occurred one week before the onset of numbness, and the brother had recently recovered from a sore throat. The neurologic examination revealed normal optic discs and cranial nerves, mild distal weakness $(4 / 5)$ in the right hand and foot, patchy loss of vibration and temperature sensation below T10, normal proprioception and pinprick sensation, negative Romberg test, mild dysmetria of the left hand, and an impaired tandem and antalgic gait. Symptoms resolved over several weeks without therapy. MRI of brain and spinal cord on admission showed lesions on T-2 weighted imaging at $\mathrm{C} 4-\mathrm{C} 5$ and $\mathrm{T} 7-\mathrm{T} 8$, and patchy hyperintensities in the white matter, especially the centrum semiovale and left cerebellum. Coronavirus OC43 was detected in CSF and nasopharyngeal secretions by PCR, and antibody titers rose from 1:160 in acute serum to 1:640 in convalescent serum at 3 weeks. Tests for other viruses were negative. CSF showed 10 red cells and 38 white blood cells, $92 \%$ lymphocytes, protein $40 \mathrm{mg} / \mathrm{dL}$, and glucose 58 $\mathrm{mg} / \mathrm{dL}$. Immunoglobulin $\mathrm{G}$ index was 0.77 (normal $<0.70$ ). A test for oligoclonal bands was omitted. MRI at 6 weeks showed improvement, but follow-up MRI at 3 months revealed a new asymptomatic lesion in the left cerebellar hemisphere, and periventricular lesions in the right cerebral hemisphere were brighter and larger. ADEM was the presumed diagnosis, the first reported case associated with coronavirus in a child, but multiple sclerosis could not be ruled out. (Yeh EA, Collins A, Cohen ME, et al. Detection of coronavirus in the central nervous system of a child with acute disseminated encephalomyelitis. Pediatrics January 2004;113:e73-e76). (Respond: E Ann Yeh MD, Women's and Children's Hospital of Buffalo, Department of Neurology, 219 Bryant St, Buffalo, NY 14222). 
COMMENT. ADEM is a post- or para-infectious illness that is usually preceded by a febrile upper respiratory viral infection (URI) or vaccination, and follows a monophasic course with recovery in $90 \%$. ADEM is distinguished from MS by long-term clinical followup, absence of relapsing course and new MRI lesions, and absent oligoclonal bands in the CSF (see Ped Neur Briefs Nov 2002;16:81-82, for commentary on long-term study of 84 children with $\mathrm{ADEM}$ ). Coronavirus ( $\mathrm{HCoV})$, a common cause of URI, has not previously been associated with ADEM, although a chronic demyelinating disorder resembling MS occurs with $\mathrm{HCoV}$ infection in the mouse model. HCoV RNA has been demonstrated in the CSF and brain of MS patients, but also in autopsy specimens of controls without neurologic disease. The role of coronavirus in demyelinating disease is undetermined.

\section{VARICELLA ZOSTER VIRUS OPTIC NEURITIS}

A 13-year-old male presented with a corticosteroid-sensitive, varicella zoster virus (VZV)-related rapid loss of vision and bilateral disc edema after a febrile illness, and the case is reported from the University of Pittsburgh School of Medicine, Pittsburgh, PA. Increasingly slow and prolonged corticosteroids taper was required to avoid relapse of visual function over a 1 year period. VZV seroconversion occurred late in the course of the disease. Neuroimaging was unrevealing. CSF was under increased pressure $(260 \mathrm{~mm} \mathrm{H} 2 \mathrm{O})$, protein $60 \mathrm{mg} / \mathrm{dL}$, glucose $48 \mathrm{mg} / \mathrm{dL}$, WBC $108 \mathrm{~mm} 3$ ( $84 \%$ lymphocytes), absent oligoclonal bands, IgG index $0.65(\mathrm{~N}<0.7)$, and polymerase chain reaction for other viruses was negative. Initial treatment with acetazolamide was unsuccessful. When prednisone was substituted, disc edema slowly improved and visual acuity became normal. Tapering of prednisone after 6 weeks was followed by recurrence of disc edema and visual loss, MRI showed T2-signal hyperintensities in the right insular and frontal cortex regions, and in the upper cervical cord. The serum and CSF VZV complement fixation assays were now positive. Treatment with intermittent steroids and oral acyclovir was followed by remission and relapse over an 18month period. At 1 year, the dose of prednisone was $20 \mathrm{mg}$ every other day, visual acuity was $20 / 20$, color perception $80 \%$, but optic discs were pale and pupil responses were defective. Otherwise, the neurologic exam was normal. (Pless ML, Malik SI. Relapsingremitting, corticosteroid-sensitive, varicella zoster virus optic neuritis. Pediatr Neurol Nov 2003;29:422-424). (Respond: Misha L Pless MD, Director, Neuro-Ophthalmology; Eye and Ear Institute, 203 Lothrop St, Pittsburgh, PA 15213).

COMMENT. VZV may cause neurologic disease without a rash (zoster sine herpete), and complications associated with viral reactivation include optic neuritis, aseptic neuritis, myelitis, and encephalitis. Immunological mechanisms are considered likely. Since seroconversion to VZV occurred within 2 months of onset in the above case, VZV was considered the most likely cause of the optic neuritis, and multiple sclerosis or ADEM was a less plausible explanation.

VZV is a human herpes virus that causes varicella as a primary infection after which the virus lies dormant in the trigeminal and dorsal root ganglia. With reactivation, the virus causes herpes zoster, sometimes followed by post-herpetic neuralgia. In a review of advances in neurological infectious diseases, Kennedy PGE (Lancet Neurology Jan 2004;3:13) cites a case of zoster sine herpete in a woman with a 13 month history of right maxillary trigeminal 